\title{
Why Do Patients Still Have the Potential to Transmit COVID-19 Despite Receiving Vaccinations?
}

\author{
Huang Wei Ling* \\ Infectious Diseases, General Practice, Nutrition, Acupuncture and Pain Management, Medical Acupuncture and Pain Management Clinic, Franca, São
} Paulo, Brazil

*Corresponding author: Huang Wei Ling, Infectious Diseases, General Practice, Nutrition, Acupuncture and Pain Management, Medical Acupuncture and Pain Management Clinic, Rua Homero Pacheco Alves, 1929, Franca, São Paulo, Brazil, Tel: +55 16 $3721-2437 ;$ E-mail: weilingmg@gmail.com

Received: 15 Sep, 2021 | Accepted: 25 Oct, 2021 | Published: 07 Nov, 2021

Citation: Huang WL (2021) Why Do Patients Still Have the Potential to Transmit COVID-19 Despite Receiving Vaccinations? Int J Vaccine Immunizat 5(1): dx.doi.org/10.16966/2470-9948.127

Copyright: @ 2021 Huang WL. This is an open-access article distributed under the terms of the Creative Commons Attribution License, which permits unrestricted use, distribution, and reproduction in any medium, provided the original author and source are credited.

\section{Introduction}

The author has written this article based on studies carried out by Mayo Clinic infectious diseases specialists. The studies stated that even people that have been vaccinated may still be carrying the virus in the nose and throat and can still potentially be a transmitter of the SARS-CoV-2 virus even when asymptomatic [1].

Perhaps this is not easy to explain in the eyes of doctors and scientists in modern Western Medicine but if we refer to ancient medicines and perhaps let go of the mentality that exists in modern medicine today we can find some of the answers [2].

According to Hippocrates, natural forces within us are the greatest healers of disease [3].

\section{What are these energies that we possess that are not curing our disease?}

In the article written by Huang (2021) entitled is "SARS-CoV- 2 Strong or Our Body Is Weak?", the author demonstrates that presently the Zheng-Qi energy is weak in the majority of the population (which is the energy that fights against the invasion of external pathogenic factors invasion). In this study, the author demonstrates that people are being colonized and infected by SARS-CoV-2 due to the low energy inside the internal massive organs, mainly the energy in the Kidney meridian (second chakra) that protects the individual against the invasion of external pathogenic factors. This lack of energy can predispose the individual to the formation of internal Heat that could be facilitating the adherence of the bacteria in the cells allowing their colonization and infection, as the author demonstrated in other publications written by her [4].

According to the article published by Huang (2021) entitled "Energy Alterations and Chakras Energy Deficiencies and Propensity to SARS-CoV-2 Infection", the author conducted a study between 2015 and 2020 and demonstrated that $90 \%$ of her patients are without energy in the five internal massive organs (that are responsible for maintaining health) which the author demonstrated through several of her published studies. Today these deficiencies are responsible for the development of infectious and non-infectious diseases such as diabetes, myocardial infarction, stroke, cancer, depression, anxiety, etc. and also for the COVID-19 pandemic that is affecting non high risk patients. According to the study, all kinds of patients have the risk of acquiring the SARS-CoV-2 infection today including babies, children, adolescences, adults and older people due to this low energy pattern. Today all chronic pathologies are based on the individual's lack of energy in their chakras energy centers [5].

In another article written by Huang (2021) entitled "What have behind in all kinds of infections that we need to know?", the author said that all bacterial, viral and fungal infectious diseases have in common chakras energy centers deficiency in energy and formation of internal Heat [6].

In the article written by Huang (2019) entitled "How Can We Treat Recurrent Herpes Virus Infection without the Use of Antiviral Drugs?", the author describes that the symptoms presented in patients with herpes (simplex HSV-1; HSV-2 or zoster) are only a reflection of energy deficiencies in the internal massive organs (that correspond to the lack of energy in the chakras energy centers identified in this article) and the formation of internal Heat. The patient's diet can be changed to rebalance the five massive internal organs by avoiding foods that could cause more formation of internal Heat (such as fried foods, eggs, chocolate, honey, coconut, melted cheese, alcoholic beverages). In addition using acupuncture associating with apex ear bloodletting and replenishing the chakras energy centers with highly diluted medications such as homeopathies (according to the theory created by Huang (2020) entitled "Constitutional Homeopathy of the Five Elements based on Traditional Chinese Medicine") can lead to the disappearance of the herpes infection symptoms without any recurrence and without using any kind of anti-viral medication. If the patient starts to resume poor eating habits or has emotional issues such as excessive anger a recurrence of the herpes problem can reoccur (due to energy imbalances in the energy of the Liver that leads to an increase in the Yang energy and formation of internal Fire) [7]. 
In the article written by Huang (2019) entitled "Why Do Patients Still Catch Hospital Infections Despite the Practice of Infection Prevention and Control Programs", the author explains that the majority of measures to control hospital infections currently are in the leaf level of the tree (that is the metaphor used by the author to explain the different viewpoints between Western medicine and traditional Chinese medicine). According to traditional Chinese medicine the emotional and physical symptoms manifested by each patient is only the reflection of energy imbalances that exists at the root of the tree (that is invisible to the naked eyes and corresponds to the two main theories in TCM that is the Yin and Yang theory and the second the Five Elements theory). Also there are the influences of the external pathogenic factors that influence the beginning to the formation of diseases mainly the infectious diseases as demonstrated by Huang (2020) in the article "Invasion of Wind and Cold as Cause of Respiratory Tract Infection Outbreak in a School Kindergarten Group of Kids" [8,9].

In another article written by Huang (2019) entitled "Is It Possible to Treat Community-acquired and Nosocomial Infectious with the Same Method without the Use of Antibiotics?", the author is showing through this article that most patients who have infection have internal energy deficiency and that the infection process is only the manifestation of internal Heat formation in these patients generated by a total energy deficiency pattern in both community and hospital infection patients [10].

In this article the author shows through some clinical cases of community and hospital infection patients that patients being infected with multi resistant bacteria can be healed through the process of removing internal Heat and rebalancing of their internal energies through Chinese dietary therapy, acupuncture, apex ear bloodletting (rebalancing the internal energy of Yin, Yang, Qi and Blood) which makes the adherence of bacteria to the tissue no longer possible. It was possible to treat the majority of these infections without using any antibiotics or antiviral medications. Often it is necessary to replenish the internal energy of the five massive organs (Liver, Heart, Spleen, Lung and Kidney) such that the individual's internal energies are strengthened thus increasing their immunity and increasing their Zheng-Q $i$ which is the internal energy responsible for the protection that fights against the entry of external pathogenic factors inside the bodies of the patient. By improving their internal energy, there will be less formation of the internal Heat that is responsible for the formation of infections and adherence of bacteria and other microorganisms in the patient's cells.

This mechanism of adherence of bacteria was written by Huang (2020) in the article "Energies Alterations and Chakras Energies Deficiencies as the Main Cause of Recurrent Urinary Tract Infection Resistant to Antibiotics Treatments". In this article the author is demonstrating that she treated patients without using any antibiotics.

In this article, the author demonstrated a patient with a history of recurring urinary tract infections for over 20 years showed no improvement after using many kinds of antibiotics. Through the treatment of:

- Rebalancing the internal energy using Chinese dietary counseling,

- Auricular acupuncture with apex ear bloodletting,

- And the replenishment of the internal massive organs energy using homeopathy medications, the patient was healed.

The theory "Constitutional Homeopathy of the Five Elements Based on Traditional Chinese Medicine" was a very important tool used by Huang to eliminate the internal energy imbalanced factors which was causing the adherence in the patient's Bladder cells, causing the urinary tract infections even after the use of antibiotics. In these cases, the use of antibiotics was causing the aggravation of internal energy deficiency which led to more internal Heat formation and this aggravated or perpetuated the symptoms found in patients with recurrent urinary tract infections $[11,12]$.

When the treatment of the infection was only focused on "killing" the bacteria or virus, the treatment itself caused a greater weakening of the patient's internal energy and continued to maintain the root energy imbalance that was keeping the adhesion of the bacteria or virus in place. Use of antibiotics maintained the conditions for bacteria or virus colonization and the potential for continued infection still existed $[8,11]$.

Therefore, where the current practice in which Western physicians tend to prescribe highly concentrated medications for the treatment of the SARS-CoV-2 infection, often without proof of efficacy and with the only aim of "killing" the virus, the effect of the medication itself is causing a weakening of the person's internal energy (according to Arndt Schultz Law) and the formation of more internal Heat. This results in the adhesion of bacteria and viruses to the cells and maintaining the process of colonization and infection. By the use of these kinds of medications (highly concentrated medications) in the treatment of this infection (SARS-CoV-2) and in the treatment of the majority of diseases today, we are reducing the vital energy of the patient (who's energy is already low due to influences of the electromagnetic waves in our human body and this leads to an immune depressed state as demonstrated by Huang (2021) in the article entitled "Are We Vaccinating Immunocompetent or Immunocompromised People for COVID 19?"). In other words, we can say that when physicians are using COVID-19 vaccines to control the pandemic, the physicians are not treating the energy deficiencies but only trying to induce the formation of antibodies in an already weakened body. This treatment is still inducing internal Heat in all of the patients because they are still very weak in energy. This leads to a propensity of the virus to adhere to the nasal mucosa and throat which allows the dissemination of the virus to others. Even asymptomatic carriers can be disseminators of the virus [13].

In an article written by Huang (2021) entitled "What We Need to Do to Improve Our Immune System Despite the Use of Vaccination for COVID-19?", the author describes the need to treat the energy deficiencies of the population in the globe. The entire population that is taking COVID-19 vaccines today may be immune deficient due to chronic exposure to electromagnetic radiation (by the use of cell phones and computers) that a large part of the world's population is exposed. This article was published by Huang (2021) entitled "The Influence of Cell Phones and Computers on Our Immune System" [14].

Since the majority of the world population is immunodeficient in order to try to reduce the transmissibility of the virus, we must improve the immunity of the general population and make them immunocompetent. Because as shown in the article written by Abbasi (2021) entitled "Researchers Tie Severe Immunosuppression to Chronic COVID-19 and Virus Variants", the author studied patient's colonization by SARS-CoV-2. Abbasi studied the patients at 23 sampling periods and discovered that the virus was adapting to the treatments and that the patient was likely to be contagious during this entire period of the study. And in this study Abbasi showed that patients with severely weakened immune systems could take months to clear the novel coronavirus and the patients could be contagious 
for an even longer time. In this article they believe that SARS-CoV-2 variants circled first among the immune compromised patients [15].

In the article written by Fernandes, et al., (2021) entitled "COVID-19 Vaccine", it was stated that the SARS-CoV-2 genome was sequenced and they used SARS-CoV-1 and SARS-CoV-2 similarities to accelerate vaccine production in the phase 3 . Current vaccines today induce immune response to the spike proteins that normally wrap the cells surface receptors to enter in the host cells. According to this article, vaccines are not infectious and do not incorporate in the host genome [16].

As you can see the COVID-19 vaccine does not have the potential to improve a person's immunodeficiency as it is injecting part of the viral genome to stimulate antibody production; using mRNA that is a novel approach in the vaccines development to generate antibody to protect the individual against SARS-CoV-2 infection [16].

In the article written by Huang (2021) entitled "Are the Vaccines the Only Solution to Control COVID-19 Pandemic?", the author is saying that due to energy deficiencies in the five internal massive organs that the majority of the population could be suffering from the organ that is responsible for the production of Blood and white cells are the Spleen (fifth chakra) and in another article written by Huang (2021) entitled "Are We Vaccinating Immunocompetent or Immunocompromised People for COVID-19?", the author is questioning if the production of antibodies in all these persons will even be effective because in this study she demonstrated that $97 \%$ of her patients did not have any energy in the fifth chakra which is responsible for the production of Blood and white blood cells $[13,17]$.

This may be a factor as to why we are having cases of the COVID-19 infection in people who had already received the AstraZeneca, Pfizer, Corona vaccine and Johnson vaccine and that most studies say that all of these post vaccine infections may be due to infections by a mutant strain. But in my own daily medical practice, I suspect that it may be due to the SARS-CoV-2 strain itself without mutant strain, due to the low antibody production in my patient pool as my patients are considered immune deficient due to their low energy pattern [17].

In the article written by Brown, et al., (2021) entitled "Outbreak of SARS-CoV-2 Infections, Including COVID-19 Vaccine Breakthrough Infections, Associated with Large Public Gatherings" - Barnstable County, Massachusetts, July 2021, the authors are demonstrating that in Massachusetts there was a vaccination coverage of $69 \%$ of the population and during a large public gathering there was a COVID-19 outbreak of three quarters of the fully vaccinated persons (who completed two doses of the Pfizer or Moderna vaccine or a single dose of Jansen vaccine). It was found that $89 \%$ of these infections were caused by the Delta variant and $1 \%$ of these infections were caused by the Delta sub lineage (A.Y.3). Among these persons 79\% were symptomatic of which five were hospitalized and four were found to be fully vaccinated. For this reason the CDC continue to explain that patients fully vaccinated still need to wear masks in indoors public settings and in areas where COVID-19 transmission is high [18].

The production of mutant's strains always occurs when the infection is occurring in immunocompromised people. Today this corresponds to the majority of the world's population according to the author. For this reason, to improve our immunity we must first act on the agents that are causing the lowering of immunity in the population. That is the influences of electromagnetic radiation across our planet [17].

While this problem cannot be resolved by our competent authorities (the influence of electromagnetic radiation in our lives); to improve the immunity of each individual on this planet, we have to protect our population by improving the production of the internal energy in our organs which are important for the maintenance of life. One of the ways to do this is to use medicines that have the potential to improve the vital energy which according to the Arndt Schultz law are highly diluted medicines according to the theory created by Huang (2020) entitled "Constitutional Homeopathy of the Five Elements Based on Traditional Chinese Medicine", as opposed to using highly concentrated medicines which further reduce the vital energy of our patients, which is already low, according to studies presented and published by the author as in the article written by Huang (2021) entitled "Energy Alterations and Chakras Energy Deficiencies and Propensity to SARS-CoV-2 Infection" [5,12].

Even if the population takes the third, fourth dose of vaccine, if the cause of the non adequate antibody formation is not treated, that the lack of energy in the chakras energy centers, they will be still immunosuppressed and not immunocompetent to suppress the viral transmission $[13,17]$.

In Japan, the government even banned Astrazenica vaccination and even donated millions of doses to other countries due to the formation of clots in some people who were taking this vaccine. In the article written by Ledford (2021) entitled "How could a COVID vaccine cause blood clots?", scientists race to investigate they are affirming that there are cases of blood clot after receiving this vaccine but scientists do not know yet what is the relation between the injection of vaccine and he formation of Blood clot. In this article, they are studying the possible causes related to the vaccine component or that is additive in the vaccine or in the vaccines production. As said by hematologist of Medical University in Vienna, Sabine Eichinger, "she does not know" [19].

The increase in cases of infection that is happening in Israel is causing the government to accelerate the application of the third dose of the vaccine against COVID-19. In the article written by Muhsen and Cohen (2021) entitled "COVID-19 vaccination in Israel", they are saying that at the end of June and beginning of July 2021, there were increasing in infection in the vaccinated individuals most of them caused by the $\delta$ variant. And they affirm in their study that the results of vaccination were less in immune compromised patients [20].

In all these studies, they are reinforcing the necessity of vaccinating the population but they are not studying the type of population that we have nowadays. According to Hippocrates ( 460 bce - 375 bce), we need to treat the patient and not the disease. According to the article written by Huang (2021) entitled "Is the Population in the World the same as in the Past?", the author is saying that the population that we have nowadays is very different from the population that we had 7 years ago, due to the different energy pattern (less energy in the internal massive organs) of our population leading to a state of general immune suppression [3,21].

Due to the lack of general energy of the world population, as demonstrated by the author in the article "Is the Population in the World the Same as in the Past?", if you return to the theme of this article that is why people are eliminating viruses that can be transmitted to others even when receiving the COVID-19 vaccine, this can be explained through the study of energy imbalances present in each individual as the author showed in other previous articles and taken into account other forms of medicine such as the reasoning used by traditional Chinese medicine [11,21].

In an article written by Huang (2021) entitled "Energy Alterations and Chakras Energy Deficiencies and Propensity to SARS-CoV-2 
Infection", the author says that more than $90 \%$ of the population studied by her has no energy in the chakras energy centers. In another study Huang (2021) wrote entitled "Are We Vaccinating Immunocompetent or Immunocompromised People for COVID-19?", She showed that in $97 \%$ of people of her study there is no energy in the Kidney (second chakra) which is responsible for protecting against the invasion of external pathogenic factors (Cold, Wind, Heat, Dryness, Humidity) and SARS-CoV-2 invasion, and due to this general low energy in the five internal massive organs, we can classify most patients nowadays as being immunodepressed because energy means our immune system in traditional Chinese medicine that it is important for prevention of diseases in the future if in balanced state. So, for the purpose of reducing the transmissibility of the SARS-CoV-2 virus of these people even receiving COVID-19 vaccination, we must turn those people who are currently immunodepressed into immunocompetent and in this way, reducing the transmission of the virus even if the person has received the vaccination $[5,13]$.

In a talk from Dr. Christina Parks from the University of Michigan, $\mathrm{PhD}$ in molecular biology, she said that COVID-19 vaccines were not designed to reduce transmission but only to attenuate symptoms. [22]

Therefore, the author recommends that to reduce the colonization of SARS-CoV-2 in the nose and throat, treatment to improve the general immunity of the population even in people who have received the vaccine or in those individuals who have not received the vaccine, using highly diluted medicines such as homeopathies according to the theory created by Huang (2020) when she began to study homeopathy in Brazil (2015) entitled "Constitutional Homeopathy of the Five Elements Based on Traditional Chinese Medicine", it is an important option of treatment of all these patients nowadays. In this theory, the author is using homeopathy medications to tone each internal massive organ, that is not working properly nowadays to maintain health and all the treatments in all types of chronic diseases are related to these lack of energy in these internal organs, as showed many articles written by the author and treating the root of all diseases that are important in the formation of Yin, Yang, Qi and Blood, we can treat all kinds of disease at the same time, even the physician does not know that the patient has such symptoms [12,23-25].

The use of homeopathic medicines according to Hahnemann's (1755 - 1843) theory (created in 1796) is of great value today in the treatment of any type of infectious and non-infectious diseases. In the Hahnemann's theory of homeopathy, he said that if the physician uses one substance in an individual this substance can cause some symptoms or diseases in this person. But this substance can be the medication to treat this person (when this individual is presenting the same symptoms caused by the exposition of this substance) if used in a much diluted infinitesimal form. For this reason, in Brazil the author is using homeopathy medications to treat COVID-19 patients using homeopathy nosodes of SARS-CoV-2 virus, prepared from secretion of patients that was proved to be infected by COVID-19 and this experience was written by the author in another article written by Huang (2021) entitled "Why Homeopathy Is the Medication of Choice in The Prevention And Treatment of COVID-19 Infection?" [26].

Even if the individual receives the treatment according to Hahnemann's theory (simillimum) there will always be a need to treat after the energy deficiency in the chakras energy centers afterwards, in all patients nowadays. This way of thinking was shown in the article Huang (2020) wrote entitled "Constitutional Homeopathic of the Five Elements based on Traditional Chinese Medicine", in which the author is explaining that homeopathy used by Hahnemann is treating as in Western medicine reasoning (at the leaf level of the tree) showed by the author in the metaphor of the tree, demonstrating the different viewpoints in Western medicine and homeopathy used by Hahnemann's and comparing with traditional Chinese medicine's reasoning. The author is showing that even treating symptoms at the leaf level, the individual will still be able to keep the energy deficiencies at the root of the tree so whenever the treatment is done at the leaf level then the treatment of energy deficiencies at the root of the tree (theory of Yin and Yang and the Five Elements theory based on traditional Chinese medicine) should be done using "Constitutional Homeopathy of the Five Elements based on Traditional Chinese Medicine" [12].

In the article written by Huang (2021) entitled "Is the Mandatory Implementation of a Passport for COVID-19 Vaccine Reduce the Transmission or Not?", the author says that this type of implantation does not make sense to exist because it will not reduce the potential of virus transmission in the community in general because even if the person is vaccinated, people still have low immunity due to low general energy (which is synonymous with low immunity according to traditional Chinese medicine) and according to study published by Mayo Clinic on August 2021, in a talk by John O’Horo infectious disease doctor, he is saying that the population and all the doctors need to aware that even if the people is vaccinated, it is still carrying the virus in the nose and throat and maintain the transmission [27].

Therefore, the strategy of treating the world population today including babies, children, adults and the elderly with highly diluted medication, in order to increase the vital energy of organs, (that are currently deficient), that is leading to the quite entire globe immunodeficiency, it is a cheaper viable solution and does not require personnel trained in the use of needles and will not have serious side effects generated by the use of this type of prevention or treatment [17].

In another article written by Wooding and Bach (2020) entitled "Treatment of COVID-19 with convalescent plasma: lessons from past coronavirus outbreaks", they are saying that in previous coronavirus who are in the course of recovery could have more adequate titles of antibodies compared to patients that has less severe cases. And more high-quality studies needed to assess whether this kind of treatment could be an effective option to treat COVID-19 [28].

But even using this kind of therapy is still not improving the immune system of the patient that is still very low. [17]

If all the population in this world use the homeopathy medications to improve their energy in the five massive organs that is proved very low nowadays (leading to a state of immune depression) all the patients would have less formation of internal Heat that is the energy imbalances increasing the viral adhesion in the nasal mucosa and throat and with this kind of measurement, the treatment would reduce the transmission to the person to person contracting and transmitting SARS-CoV-2, because we will increase the immunity of the population and leading to less spread by the virus, as it occurs mostly in immune deficient group of patients, according to the study wrote by Abbasi (2021) entitled "Researchers Tie Severe Immunosuppression to Chronic COVID-19 and Virus Variants" [13,15].

As said by Hippocrates (460 bce - 375 bce), natural forces within us are the greatest healers of the disease. According to him, we must treat the patient and not the disease. So if we treat the deficiencies in the patient who is immune compromised nowadays they will certainly become stronger and be healthier in the point that their own defenses will prevent colonization and infection because according to the article 
"Researchers Tie Severe Immunosuppression to Chronic COVID-19 and Virus Variants", the author is affirming that the immunosuppressed people will keep the infection and the production of mutants. However as the author says in the article written by Huang (2021) entitled "Are We Vaccinating Immunocompetent or Immunocompromised People for COVID 19?", The proportion of immunosuppressed people is undervalued and only those who have a past history of a cancerous disease or who are using immunosuppressive medication are considered immune suppressed nowadays $[3,13,15]$.

This is explained by the way medicine is practiced today where something that are considered scientific only if can be proven through laboratory or radiological tests after the implementation of Flexner report in 1910. But if we analyze how the development of disease is seeing (the evolution from health to disease) this takes place through five phases being the first three phases, due to internal energy alterations but there are no laboratory alterations. Only in phases 4 and 5 of the disease evolution, laboratory or radiological alterations occur (but in a very advanced phase of energy imbalances). According to the article "Researchers Tie Severe Immunosuppression to Chronic COVID-19 and Virus Variants" if it is immunosuppressed people who are transmitting the infection, therefore this group of patients correspond to almost the entire world population if we proportionally make the list of immunodepressed people found in the study carried out by Huang (2021) entitled "Are We Vaccinating Immunocompetent or Immunocompromised People for COVID 19?” [8,13].

If we treat only the disease and not the patient, who remains immunosuppressed, we will not treat the cause but only the effect therefore, people would continue contracting the virus and transmitting the virus, perpetuating the transmission $[13,17]$.

\section{Acknowledgements}

Special thanks to Ronald Howard Fong, for proofreading the text grammatically and structurally.

\section{References}

1. Stiepan DD (2021) COVID-19 infection among vaccinated people: What you need to know. Mayoclinic.

2. Ling HW (2021) What Do All Autoimmune Diseases Have in Common? J Clin Exp Immunol 6: 301-304.

3. Craik EM (2014) The 'Hippocratic' Corpus Content and Context. ( $1^{\text {st }}$ edition) Routledge, Fife, UK, 344.

4. Ling HW (2021) Is SARS-CoV- 2 Strong or Our Body Is Weak? J Virol Viral Dis 1: 1-5.

5. Ling HW (2021) Energy Alterations and Chakras' Energy Deficiencies and Propensity to SARS-CoV-2 Infection. Acta Scientific Microbiology 4: 167-196.

6. Ling HW (2021) What have behind in all kinds of infections that we need to know? Journal of Investigative Oncology 1: 18-21.

7. Huang WL (2019) How Can we Treat Recurrent Herpes Virus Infection Without the Use of Antiviral Drugs? Acta Scientific Medical Sciences 3: 152-159.

8. Ling HW (2018) Why do Patients Still Catch Hospital Infections Despite the Practice of Infection Prevention and Control Programs? Acta Scientific Microbiology 1: 34-43.

9. Huang WL (2020) Invasion of Wind and Cold as Cause of Respiratory Tract Infection Outbreak in a School Kinder Garden Group of Kids. Kids. Pediatr Res Child Health 3: 1-4.
10. Ling HW (2019) Is it Possible to Treat Community-Acquired and Nosocomial Infections with the Same Method, Without the Use of Antibiotics? J Appl Microb Res 2: 1-13.

11. Ling HW (2020) Energies Alterations and Chakras Energies Deficiencies as the Main Cause of Recurrent Urinary Tract Infection Resistant to Antibiotics Treatments. Clinic Res Urol 3: 1-8.

12. Ling HW (2020) Constitutional Homeopathy of the Five Elements based on Traditional Chinese Medicine. Acta Scientific Medical Sciences 4: 57-69.

13. Huang WL (2021) Are We Vaccinating Immunocompetent or Immunocompromised People for COVID 19? J Vaccines Res Vaccin 7: 1-10.

14. Huang WL (2021) The Influence of Cell Phones and Computers on Our Immune System. Ann Immunol Immunother 3: 000141.

15. Abbasi J (2021) Researchers Tie Severe Immunosuppression to Chronic COVID-19 and Virus Variants. JAMA 325: 2033-2035.

16. Fernandes A, Chaudhari S, Jamil N, Gopalakrishnan G (2021) COVID-19 Vaccine. Endocr Pract 27: 170-172.

17. Ling HW (2021) Are the Vaccines the Only Solution to Control COVID-19 Pandemic? Vaccines Vacccin 6: 1-6.

18. Brown CM, Vostok J, Johnson H, Burns M, Gharpure R, et al. (2021) Outbreak of SARS-CoV-2 Infections, Including COVID-19 Vaccine Breakthrough Infections, Associated with Large Public GatheringsBarnstable County, Massachusetts, July 2021. MMWR 70: 10591062.

19. Ledford H (2021) How could a COVID vaccine cause blood clots? Scientists race to investigate. Nature 592: 334-335.

20. Muhsen K, Dani C (2021) COVID-19 vaccination in Israel. Clin Microbiol Infect 1-5.

21. Ling HW (2021) Is the Population in the World the Same as in the Past? Acta Scientific Clinical Case Reports 2: 70-71.

22. Park C (2021) Why Do Patients Still Have the Potential to Transmit COVID-19 despite Receiving Vaccinations?

23. Ling HW (2019) Why Are Diabetic Patients Still Having Hyperglycemia despite Diet Regulation, Antiglycemic Medication and Insulin? Int J Diabetes Metab Disord 4: 1-14.

24. Huang WL (2020) The Importance of Treating Energy Imbalances and Chakras Replenishment for Prevention and Treatment of Cancer. ACRCI 3: 1-10.

25. Ling HW (2021) What We Need to Know When the Patient has a Stroke with or without COVID-19? Acta Scientific Neurology 4: 0105.

26. Huang WL (2021) Why Homeopathy Is the Medication of Choice in the Prevention and Treatment of COVID-19 Infection. Clinical Medicine and Health Research Journal 1: 1-6.

27. Ling HW (2021) Is the Mandatory Implementation of a Passport for COVID-19 Vaccine Reduce the Transmission or Not? Acta Scientific Microbiology 4: 63-68.

28. Wooding DJ, Bach H (2020) Treatment of COVID-19 with convalescent plasma: lessons from past coronavirus outbreaks. 26: 1436-1446. 